\title{
Auto-extraction of space debris features based on autofocus search and inverse Radon transform
}

\author{
Huawei $\mathrm{Cao}^{1, \mathrm{a}}$, Meiguo $\mathrm{Gao}^{2, \mathrm{~b}}$ \\ ${ }^{1,2}$ School of Information and Electronic, Beijing Institute of Technology, Beijing 100081, P. R. China \\ aHwei.cao@gmail.com, ${ }^{\mathrm{b}}$ meiguo_g@bit.edu.cn
}

Keywords: Space debris; Feature extraction; Doppler signature; Autofocus search; Inverse Radon transform.

\begin{abstract}
A method for automatic and accurate feature extraction of space debris is proposed. The echo model of space debris in orbit is formulated first. Then an autofocus search method is presented to obtain the Doppler signature of space debris based on the predicted orbit. The inverse Radon transform (IRT) is used to automatically extract features of space debris. The extraction of spin periods is considered as an example to illustrate the effectiveness of the proposed method. Quantitative simulation results prove that estimated parameter is sufficient in accuracy.
\end{abstract}

\section{Introduction}

As it is mentioned in JFCC SPACE satellite situation report in 2015 [1], about 22\% of space targets are spacecrafts (includes satellites, space station and so on), and about $78 \%$ of space targets are space junk (includes space debris and abandoned spacecrafts). These numbers imply that it is absolutely essential to do the research on space debris identification [2]. Various kinds of space debris features are required to realize the identification of space debris [3, 4], such as shape, spin period, and so on.

At present, for the narrow-band radar, the researches on the feature extraction of space targets are mostly based on Radar Cross-Section (RCS) [5-9]. The resolution of small-sized space targets, especially space debris, is restricted by the range resolution [9]; thus, it is generally unsatisfactory to extract the feature of space debris only from the amplitude of echoes in this situation. In fact, for the narrow-band coherent radar, more features of radar targets can be extracted from the phase of echoes [10-14].

The method proposed in [13] extracts the spin period of space targets in orbit from the phase of radar echoes successfully in an user-assisted fashion. In addition, when the predicted orbit is not sufficient in accuracy, the effectiveness of this method is below expectations. Therefore, we propose an autofocus search method to compensate orbit errors and then utilize the inverse Radon transform (IRT) to automatically extract the spin period of space debris.

The rest of this paper is organized as follows: Section 2 gives the signal model. Section 3 introduces the autofocus search method and discusses the auto-extraction of space debris spin period based on the IRT. The simulation results are shown in Section 4. The conclusions are presented in Section 5 .

\section{Signal model}

The single pulse radar transmits linear frequency modulated (LFM) signals to space, and we assume that the signal model of the transmitted signal is

$$
s_{t}\left(t_{m}, t_{n}\right)=\operatorname{rect}\left(\frac{t_{m}}{T_{p}}\right) e^{j 2 \pi\left(f_{0} t_{m}+\frac{1}{2} K t_{m}^{2}\right)} e^{j 2 \pi f_{R F} t_{n}},
$$




$$
\operatorname{rect}\left(\frac{t_{m}}{T_{p}}\right)= \begin{cases}1 & -\frac{T_{p}}{2}<t_{m}<\frac{T_{p}}{2} \\ 0 & \text { otherwise }\end{cases}
$$

where $K=B / T_{p}$ is the slope of LFM, $t_{m}$ is the fast time, $t_{n}$ is the slow time, $B$ is the bandwidth of LFM signal, $T_{p}$ is the pulse width of LFM signal, $f_{0}$ is the initial frequency of LFM signal, and $f_{R F}$ is the center frequency of the transmitted signal.

The received signal is

$$
\begin{aligned}
& S_{r}\left(t_{m}, t_{n}\right)=\delta r\left[t_{m}-\tau\left(t_{m}\right)\right] e^{j 2 \pi f_{R F}\left[t_{n}-\tau\left(t_{n}\right)\right]}, \\
& r(t)=\operatorname{rect}\left(\frac{t}{T_{p}}\right) e^{j 2 \pi\left(f_{0} t+\frac{1}{2} K t^{2}\right)},
\end{aligned}
$$

where $\delta$ is the complex scatter coefficient of space target, $\tau(t)=\left(2 R_{0}-2 v t-a t^{2}\right) / c$ is the delay of radar echoes, $R_{0}$ is the radial range, $v$ and $a$ are the radial velocity and radial acceleration respectively, and $c$ is the speed of light.

After removing the carrier from the received signal, the baseband signal can be written as

$s_{r}^{\prime}\left(t_{m}, t_{n}\right)=\delta r\left[t_{m}-\tau\left(t_{m}\right)\right] e^{-j 2 \pi f_{R F} \tau\left(t_{n}\right)}$.

The substitution of these relations in (5) yields,

$$
s_{r}^{\prime}\left(t_{m}, t_{n}\right)=\delta \operatorname{rect} \frac{t_{m}-\tau\left(t_{m}\right)}{T_{p}} e^{-j 2 \pi f_{R F} \tau\left(t_{n}\right)} \cdot e^{j 2 \pi\left[f_{0}\left(t_{m}-\tau\left(t_{m}\right)\right)+\frac{1}{2} K\left(t_{m}-\tau\left(t_{m}\right)\right)^{2}\right]} .
$$

Then the signal is processed by a matched filter, and the matched filter output is

$$
s_{r c}\left(t_{m}, t_{n}\right)=\delta T_{p} \operatorname{sinc}\left[\pi K T_{p}\left(t_{m}-\tau\left(t_{m}\right)\right)\right] e^{-j 2 \pi f_{R F} \tau\left(t_{n}\right)} \text {. }
$$

The envelope shape of $s_{r c}\left(t_{m}, t_{n}\right)$ is a sinc function, and the peak position is decided by $\tau\left(t_{m}\right)$ which is related to the radial range. The exponential function in (7) contains the phase information which is modulated by the motion of space target. Because of the radial velocity and radial acceleration of space target, the signal energy spread on several range bins and different Doppler units. It is necessary to compensate the velocity error and acceleration error before the coherent integration.

\section{Auto-extraction method}

\subsection{Autofocus search}

Based on the predicted orbit of space debris, we use an autofocus search method to estimate the velocity error and acceleration error. Then subtly align the envelope and compensate the phase error of the echoes before the coherent integration. While eliminating the effect of translational component of space debris, the residual rotational component of space debris could be used to extract the characteristic. The detail of the proposed method is discussed as follows.

a. Generate sampling gates by the predicted orbit of space debris. Then collect echoes by the sampling gates and convert the frequency of the echoes down to baseband.

b. Choose appropriate search values of search parameters based on the predicted orbit of space debris.

Based on the predicted range of space debris $R_{p}(j)(j=1,2, \mathrm{~L}, N)$, generate the range search values $\hat{R}_{i, j}$ for each baseband echo which can be written as

$$
\hat{R}_{i, j}=R_{p}(j)+\hat{v}_{i}(j-1) p r t+\frac{1}{2} \hat{a}_{i}((j-1) p r t)^{2},
$$

where $\hat{v}_{i}(i=1,2, \mathrm{~L}, M)$ is the search value of radial velocity, and $\hat{a}_{i}$ is the search value of radial acceleration, prt is the pulse repetition time, $i$ represents the $i$-th search value, $j$ represents the 
$j$-th baseband echo, $M$ is the total number of search values, and $N$ is the total number of the echoes to be coherent integrated.

An appropriate value of $N$ could be calculated as

$$
N=\left\lceil\left(\frac{R_{t}}{R_{\max }}\right)^{4} \frac{\sigma}{\sigma_{t}}\right\rceil,
$$

where $R_{t}$ is the radial range of space debris, $\sigma_{t}$ is the RCS of the space debris, $R_{\max }$ is the maximum radar detection distance when the RCS of space debris is $\sigma$, and $\lceil\cdot\rceil$ represents the rounding operation.

The search values of the velocity and acceleration could be expressed by the initial values and the search steps.

$$
\left\{\begin{array}{l}
\hat{v}_{i}=\hat{v}_{0}+\Delta v(i-1) \\
\hat{a}_{i}=\hat{a}_{0}+\Delta a(i-1)
\end{array},\right.
$$

where $\hat{v}_{0}$ and $\hat{a}_{0}$ are the initial values of velocity and acceleration; $\Delta v$ and $\Delta a$ are the search steps of these two search parameters which are decided by the migration of envelopes and the spread of Doppler.

We assume that the migration of the envelope is less than the range sampling cell $L_{r}$, and the spread of Doppler is less than the Doppler cell $L_{d}$. Then the two search steps could be calculated as

$$
\left\{\begin{array}{l}
\Delta v \leq \frac{c L_{r}}{2 F_{s} N p r t} \\
\Delta a \leq \frac{\lambda L_{d}}{2(N p r t)^{2}}
\end{array}\right.
$$

where $F_{s}$ is the sampling frequency of the received signal, and $\lambda$ is the wavelength of the transmitted signal.

c. Align the envelope in a straight line by the range search parameter.

The generation of the sampling gates in step a is a pre-alignment of the envelope. Assume that $\tau_{0}$ is the start time of received signal which is decided by the distance between radar and space debris, and $\tau_{0}^{\prime}$ is the start time of sampling gate. Because of the digital sampling, $\tau_{0}^{\prime}$ is quantified by the sampling clock. Therefore, there is an error $\Delta \tau_{0}$ between $\tau_{0}^{\prime}$ and $\tau_{0}$. The delay error $\Delta \tau_{0}$ must settle for $\left|\Delta \tau_{0}\right| \leq 1 / F_{s}$, and the accurate value of the delay error can be calculated as

$$
\Delta \tau_{0}=\frac{2\left(\hat{R}_{i, j}-\left[\frac{\hat{R}_{i, j} \cdot 2 F_{s}}{c}\right] \frac{c}{2 F_{s}}\right)}{c} .
$$

According to the time shift property of discrete Fourier transform (DFT), if $x[n] \leftrightarrow X\left(e^{j \Omega}\right)$ (a couple of Fourier transform pairs), then $x[n-m] \leftrightarrow X\left(e^{j \Omega}\right) e^{-j \Omega m}$. In the digital sampling system, the delay error $\Delta \tau_{0}$ can not be expressed in time domain but can be transformed to a phase error $e^{-j \Omega \Delta \tau_{0}}$ in frequency domain. To subtly align the envelope, the frequency spectrum of the baseband signal is multiplied by the envelope alignment factorial Envelop $_{\text {com }}(i, j)$, which is

$$
\text { Envelop }_{\text {com }}(i, j)=e^{j 2 \pi F_{s} \Delta \tau_{0}} \text {. }
$$

After that the signal is processed by a matched filter in frequency domain. Then the frequency domain signal is transformed into the time domain signal.

d. Compensate the phase error. Multiply the above signal by the phase compensation factorial Phase $_{\text {com }}(i, j)$ in the time domain, which is related to the range search parameter as 


$$
\text { Phase }_{\text {com }}(i, j)=e^{j 2 \pi f_{R F} \frac{2 \hat{R}_{i, j}}{c}}=e^{j 4 \pi \frac{\hat{R}_{i, j}}{\lambda}} \text {. }
$$

e. After the envelope alignment and phase compensation, utilize fast Fourier transform (FFT) in Doppler dimension, and then record the peak value in Range-Doppler dimension.

f. Change another search value, and repeat steps c to e.

g. Find the maximum value in the recorded peak values, and record the data in Doppler dimension which are corresponding to the maximum value. The Doppler data of different time frames are ranked in order of time and then a time-frequency graph is obtained which contains the Doppler signatures of space debris.

\subsection{Feature extraction}

The Doppler of the three-axis stabilized target focuses on a single spectrum line, and it means that the phase center is stable. However, the Doppler of the roll or spin target is like a mountain or has many separate spectrum lines which is caused by the movement of multiple scattering centers [13].

When space debris spin, the scattering center of space debris will cause a sinusoid in the time-frequency plane [15]. Many papers introduce the characteristic extraction of radar target based on the IRT $[15,16]$. In this paper, the IRT is used to estimate the spin period of space debris automatically.

According to the IRT principle, a sinusoid in the time-frequency plane can be transformed into a point in the IRT plane. To integrate all energy along a sinusoid into one point, an appropriate value of the rotate angle $\hat{\theta}$ is required [15]. For the highest energy concentration, the optimal value of $\hat{\theta}$ should be $\hat{\theta}=2 \pi T / T_{\omega}$, where $T$ is the observation time, and $T_{\omega}$ denotes the spin period.

With different search values of the rotate angle, we obtain different peak values in the IRT domain. The maximum peak value corresponds to the optimal rotate angle $\hat{\theta}$. Then the value of spin period can be calculated. Note that the components with zero Doppler in the time-frequency plane will disturb the estimation of optimal rotate angle; thus, it should be eliminated by high pass filter before the IRT operation.

\section{Simulation}

The feasibility and effectiveness of the proposed method are validated by numerical simulations. Assume that there are three strong scattering centers $\left(p_{1}, p_{2}, p_{3}\right)$ on space debris with different scattering coefficients $(1,0.8,0.6)$, and the local coordinates of them are $[0,0,0],[-0.5,-0.5,-0.8]$, $[1,0.8,0.4]$ respectively. We also assume that the spin period of space debris is 20.49s. The radar system parameters are shown in Table. 1.

Table 1 The radar system parameters

\begin{tabular}{|l|l|}
\hline Parameters name & Parameters value \\
\hline Carrier frequency & $3[\mathrm{GHz}]$ \\
\hline Pulse repetition frequency & $300[\mathrm{~Hz}]$ \\
\hline Bandwidth of transmit LFM signal & $5[\mathrm{MHz}]$ \\
\hline Number of integrated pulses & 256 \\
\hline Total observation time & $50[\mathrm{~s}]$ \\
\hline
\end{tabular}




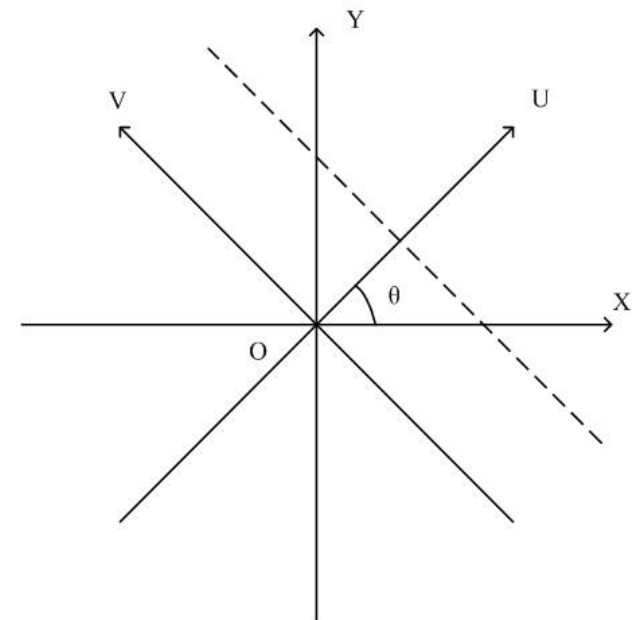

Fig.1 The time-frequency graph of the simulation data

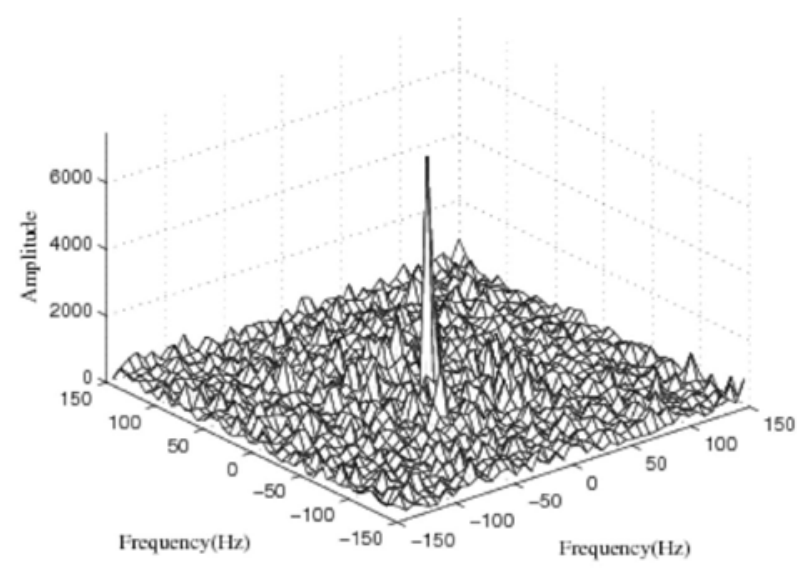

Fig.3 Peak values in the IRT domain with different search values of spin period

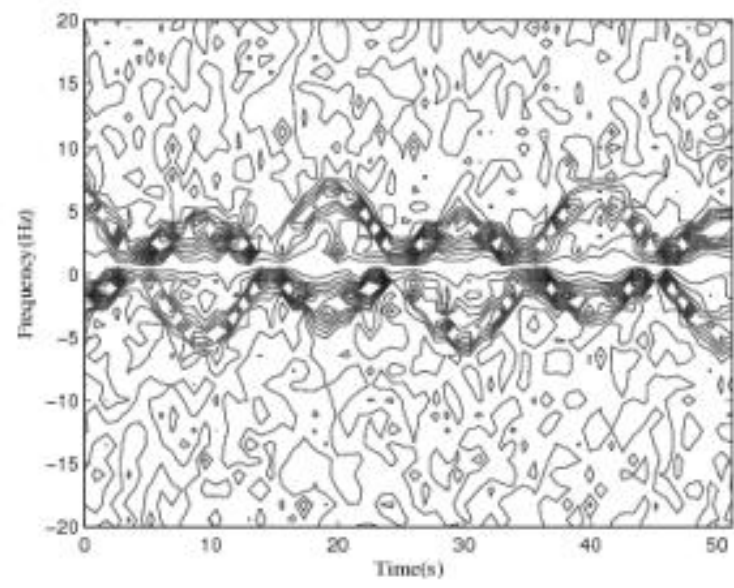

Fig.2 The time-frequency graph without zero Doppler components

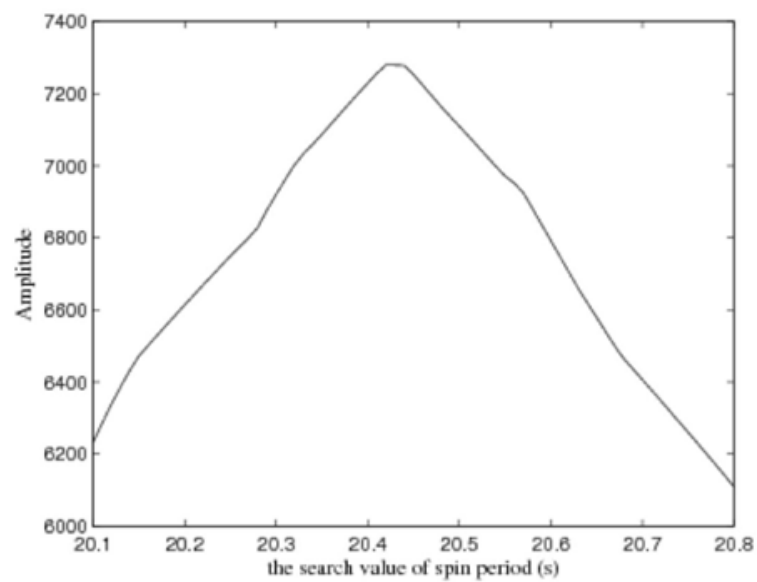

Fig.4 Inverse Radon transform with highest energy concentration (the search value of spin period is $20.42 \mathrm{~s}$ )

According to the execution steps of the proposed method discussed in section 3 , we obtain the primary results, as shown in Fig. 2. The movement of strongest scattering center $p_{1}$ causes the straight line in the time-frequency plane, and that of the scattering centers $p_{2}, p_{3}$ cause two different sinusoids. The different peak values in the IRT domain with different search values of spin period are shown in Fig. 3. The IRT plane with the highest energy concentration is figured in Fig. 3.

According to Fig. 4, the estimated spin period is $20.42 \mathrm{~s}$, and the relative error is $0.342 \%$. The simulation proves that the proposed method is appropriate to extract the spin period of space debris and the estimated parameter is sufficient in accuracy. This experiment also reveals that the present method is effective to integrate the energy of sinusoids in the time-frequency plane even when there is a stable scattering center.

In addition, based on the estimated spin period and the coordinates of the peak in Fig. 4, it is possible to estimate the shape of space debris [15].

\section{Conclusion}

In this paper, an automatic method for features extraction of space debris based on the autofocus search algorithm and the inverse Radon transform is presented. The simulation results prove that the proposed method is feasible to extract the feature of space debris in orbit from the phase of radar echoes. The presented auto-extraction method can be generalized to extract the shape of space debris with a fast spin rate, which is our future work. 
[1] JFCC SPACE. The Satellite Situation Report. 2015-07-23. https://www.space-track.org.

[2] B. M. Kent, C. Thomas, and P. Ryan: The nasa debris radar for characterizing static and dynamic ascent debris events for safety of flight. IEEE Antennas and Propagation Society International Symposium (APSURSI), (2012),p. 1-2.

[3] D. Mehrholz, L. Leushacke and W. Flury, in: Detecting, tracking and imaging space debris. ESA Bulletin (0376--4265), Vol. 109 (2002), p. 128-134.

[4] Z. J. Dai, W. X. Yu and W. D. Hu, in: Techniques of Radar Recognition for Space Targets. Systems Engineering and Electronics, Vol. 22 (2000): 19-22.

[5] N. Rajan, T. Morgan and R. Lambour: Orbital debris size estimation from radar cross section measurements. Proc. of the 3rd European Conference on Space Debris, (2001), p 83-88.

[6] S. Chan and K. Lee. Radar target identification by kernel principal component analysis on rcs. Journal of Electromagnetic Waves and Applications, Vol. 26 (2012), p. 64-74.

[7] X. H. Lei, X. J. Fu and C. Wang, in: Statistical feature selection of narrowband rcs sequence based on greedy algorithm. Proc. of the 2011 IEEE CIE International Conference on Radar, Vol. 2 (2011), p.1664-1667.

[8] X. H. Xiao, Y. Ma and Z. P. Chen, in: Research on size of space object estimated from RCS. Signal Processing, Vol.6 (2005), p.639-641.

[9] C. Wang, X. J. Fu and Q. Zhang, in: Space object identification based on narrowband radar cross section. Proc. of the 5th International Congress on Image and Signal Processing, (2012), p. 1653-1657.

[10] K. L. Li, W. D. and Jiang, X. Li. in: Micro-motion feature analysis and extraction methods for ballistic targets. Systems Engineering and Electronics, Vol. 32 (2010): p. 115-118.

[11] I. Bilik,J. Tabrikian, in: Radar target classification using doppler signatures of human locomotion models. IEEE Transactions on Aerospace and Electronic Systems, Vol. 43 (2007), p. 1510-1522.

[12] T. Sato. Shape estimation of space debris using single-range doppler interferometry. IEEE Transactions on Geoscience and Remote Sensing, Vol. 37 (1999), p. 1000-1005.

[13]D. F. Chen and M. G. Gao, in: Characteristic analysis of space targets in orbit based on the phase of radar echoes. Microcomputer Information, Vol. 28 (2012).

[14] Y. Luo, Q. Zhang and C. W. Qiu, in: Micro-Doppler effect analysis and feature extraction in ISAR imaging with stepped-frequency chirp signals. IEEE Transactions on Geoscience and Remote Sensing, ,Vol. 48 (2010), p. 2087-2098.

[15] H. H. Yan, X. J. Fu and P. Li, in: Detection and measurement to multiple scatterers with micro-motion based on inverse radon transform. Transactions of Beijing Institute of Technology, Vol.32 (2012), p.526-530.

[16] M. Dakovic and L. Stankovic, in: Estimation of sinusoidally modulated signal parameters based on the inverse radon transform. Proc. of the 8th International Symposium on Image and Signal Processing and Analysis (ISPA), (2013), p. 302--307. 